BMJ Open

Diabetes

Research

\& Care

\title{
DNA methylation analysis of cord blood samples in neonates born to gestational diabetes mothers diagnosed before 24 gestational weeks
}

\author{
Yoshifumi Kasuga, ${ }^{1,2}$ Tomoko Kawai (1) , ${ }^{3}$ Kei Miyakoshi, ${ }^{1}$ Asuka Hori, ${ }^{2,4}$ \\ Masumi Tamagawa, ${ }^{1}$ Keita Hasegawa, ${ }^{1,2}$ Satoru Ikenoue, ${ }^{1}$ Daigo Ochiai, ${ }^{1}$ \\ Yoshifumi Saisho (1) , Mariko Hida, ${ }^{6}$ Mamoru Tanaka, ${ }^{1}$ Kenichiro Hata ${ }^{2}$
}

\begin{abstract}
To cite: Kasuga Y, Kawai T, Miyakoshi K, et al. DNA methylation analysis of cord blood samples in neonates born to gestational diabetes mothers diagnosed before 24 gestational weeks BMJ Open Diab Res Care 2022:10:e002539. doi:10.1136/ bmjdrc-2021-002539
\end{abstract}

Received 13 August 2021 Accepted 3 December 2021

Check for updates

C) Author(s) (or their employer(s)) 2022. Re-use permitted under CC BY-NC. No commercial re-use. See rights and permissions. Published by BMJ.

For numbered affiliations see end of article.

Correspondence to Kenichiro Hata; hata-k@ncchd.go.jp and Dr Tomoko Kawai; kawai-tm@ncchd.go.jp

\section{ABSTRACT}

Introduction Genome-wide methylation analyses of gestational diabetes mellitus (GDM) diagnosed after 24 gestational weeks (late GDM (L-GDM)) using cord blood have been reported. However, epigenetic changes in neonates born to mothers with GDM diagnosed before 24 gestational weeks (early GDM (E-GDM)) have not been reported. We investigated DNA methylation in neonates born to mothers with E-GDM using cord blood samples. Research design and methods Genome-wide DNA methylation analysis was performed using an Illumina EPIC array to compare methylation rates of 754255 autosomal sites in cord blood samples from term neonates born to 162 mothers with GDM (E-GDM: $n=84$, L-GDM: $n=78$ ) and 60 normal glucose tolerance (normal OGTT) pregnancies. GDM was diagnosed based on Japan Society of Obstetrics and Gynecology criteria modified with International Association of Diabetes in Pregnancy Study Group criteria. In this study, all GDM mothers underwent dietary management, while self-monitoring of blood glucose and insulin administration was initiated when dietary modification did not achieve glycemic control.

Results There were no significant differences in genomewide DNA methylation of cord blood samples between the GDM (E-GDM and L-GDM) groups and normal OGTT group or between the E-GDM and normal OGTT groups, L-GDM and normal OGTT groups, and E-GDM and L-GDM groups. Conclusions This is the first report to determine the DNA methylation patterns in neonates born to mothers with E-GDM. Neonates born to mothers with GDM, who were diagnosed based on Japan Society of Obstetrics and Gynecology criteria, may not differ in DNA methylation compared with those born to normal OGTT mothers.

\section{INTRODUCTION}

Gestational diabetes mellitus (GDM) is a common perinatal complication associated with poor prognosis during the perinatal period (ie, cesarean section (CS), shoulder dystocia, macrosomia (birthweight $\geq 4000 \mathrm{~g}$ ), and neonatal hypoglycemia or jaundice) and the next generation's future healthcare (ie, obesity and metabolic syndromes) ${ }^{1-3}$ In recent reports on GDM diagnosed after 24 gestational

\section{Significance of this study}

What is already known about this subject?

- The intrauterine environment in maternal gestational diabetes mellitus (GDM) is reportedly associated with changes in fetal DNA methylation.

What are the new findings?

- There was no significant association between cord blood DNA methylation values and GDM group compared with that in the normal oral glucose tolerance test (OGTT) group.

- There was no significant association between cord blood DNA methylation values and GDM diagnosed before 24 gestational weeks (early GDM (E-GDM)) or GDM diagnosed after 24 gestational weeks (late GDM (L-GDM)) group compared with that in the normal OGTT group.

- There was no significant association between cord blood DNA methylation values between cord blood DNA methylation values and the E-GDM group compared with the L-GDM group.

- There was no significant difference in cord blood DNA methylation level in GDM with insulin and diet therapy group and GDM with only diet-controlled group.

How might these results change the focus of research or clinical practice?

- Since we found no significant differences in umbilical cord blood DNA methylation between E-GDM or L-GDM and normal OGTT, from a methylation analysis perspective, appropriate treatment for GDM may defend fetal epigenetic changes.

weeks (late GDM (L-GDM)), epigenetic modifications associated with maternal hyperglycemia were assumed to mediate the susceptibility to metabolic disorders in offspring. The intrauterine environment in maternal GDM was reportedly associated with changes in fetal DNA methylation at CpG (5'-Cytosinephosphate-Guanine-3) sites in metabolic 
genes. ${ }^{4-12}$ Based on these studies, the mechanisms of future metabolic syndrome and obesity development were evaluated from an epigenetic perspective. However, these studies were based on small cohorts and did not characterize the maternal glycemic status during pregnancy nor provided detailed fetal/neonatal information.

GDM has been diagnosed and managed before 24 gestational weeks (early GDM (E-GDM)) in Japan. ${ }^{13}$ While it was reported that effectiveness of therapeutic intervention for E-GDM was lower than that for L-GDM, ${ }^{14}$ recently, the importance of treatment for E-GDM is becoming increasingly recognized. ${ }^{15}$ Because women with E-GDM show higher frequencies of preterm birth than those diagnosed with L-GDM, and often exhibit poor prognosis (ie, pre-eclampsia, CS, admission to neonatal intensive care units, macrosomia or large for gestational age (birthweight $\geq 90$ th percentile) and neonatal jaundice) ${ }^{16-19}$ However, it is not known how therapeutic interventions for E-GDM affect epigenetic change in offspring.

The aim of this study was to investigate genomewide methylation in cord blood from offspring born to mothers with GDM, particularly those mothers with E-GDM, through DNA methylation analysis.

\section{MATERIALS AND METHODS}

\section{Study population}

Umbilical cord blood samples were collected from single-term neonates born to Japanese mothers with GDM ( $n=167)$ or normal glucose tolerance (normal oral glucose tolerance test (OGTT); $\mathrm{n}=63$ ) and who received perinatal care at Keio University Hospital (Tokyo, Japan) from January 2011 to December 2016. In our hospital, as described in our previous report, women who screened positive for GDM in the first trimester had at least one of the following criteria: random plasma glucose level $\geq 95 \mathrm{mg} / \mathrm{dL}$, hemoglobin A1c $\geq 5.9 \%$, glycoalbumin $\geq 15.8 \%$, a personal history of GDM or macrosomia delivery, family history of type 2 diabetes, or prepregnancy body mass index (BMI) $\geq 25 \mathrm{~kg} / \mathrm{m}^{2}$. When patients were positive for E-GDM screening, they immediately underwent a $75 \mathrm{~g}$ OGTT to diagnose E-GDM. ${ }^{15}$ As a two-step procedure to diagnose L-GDM is used in Japan, women who screened negative or had normal OGTT results in the first trimester were also evaluated by a $50 \mathrm{~g}$ glucose challenge test at 24-28 gestational weeks. If the glucose challenge test result was positive $(\geq 7.8 \mathrm{mmol} / \mathrm{L}$ $(140 \mathrm{mg} / \mathrm{dL}))$, the mothers immediately underwent the diagnostic $75 \mathrm{~g}$ OGTT. According to the additionally modified International Association of Diabetes in Pregnancy Study Group (IADPSG) criteria by Japan Society of Obstetrics and Gynecology, E-GDM and L-GDM were diagnosed when a pregnant woman exceeded one or more of the following thresholds: fasting plasma glucose (FPG) of $92 \mathrm{mg} / \mathrm{dL}$, OGTT 1-hour plasma glucose (PG) of $180 \mathrm{mg} / \mathrm{dL}$, OGTT 2-hour plasma glucose of $153 \mathrm{mg} /$ dL. Each subject was diagnosed based on the OGTT, and all mothers with GDM underwent dietary management and self-monitoring of blood glucose measurements at our institution, with evaluations performed every 2 or 3 weeks. Insulin administration was initiated when dietary treatment did not achieve the glycemic goal. In this study, women who screened as negative for OGTT at 24-28 gestational weeks were defined as the normal OGTT group. ${ }^{15}$ Exclusion criteria included multiple pregnancies, preterm birth (gestational age at delivery $<37$ weeks), fetal growth restriction, hypertensive disorder during pregnancy, neonatal asphyxia (umbilical artery $\mathrm{pH}<7.1$ or Apgar score at $5 \mathrm{~min}<7$ ), and congenital fetal anomaly. Additionally, we excluded women with diabetes mellitus (ie, type 1 or 2) before pregnancy and overt diabetes during pregnancy, with the latter defined as hemoglobin A1c $>6.5 \%, F P G \geq 126 \mathrm{mg} / \mathrm{dL}$, or random $\mathrm{PG}$ level $\geq 200 \mathrm{mg} / \mathrm{dL}$ and requiring confirmation by one of the first two measures. Insulin sensitivity and secretion were evaluated based on parameters calculated using the diagnostic $75 \mathrm{~g}$ OGTT. The insulin sensitivity was evaluated using a whole-body insulin sensitivity index derived from the OGTT ( $\mathrm{IS}_{\mathrm{OGTT}}$ ) and homeostasis model assessment of insulin resistance (HOMA-IR) ${ }^{20}{ }^{21}$ Insulin secretion was assessed based on the insulinogenic index, ${ }^{22}$ and beta-cell function was calculated as the OGTT-derived disposition index using insulin secretion sensitivity index (ISSI) $2 .^{23}$

DNA methylation analysis using umbilical cord blood samples The methods of DNA methylation analysis was described in our previous report. ${ }^{24}$ Umbilical cord blood was collected from each neonate immediately after birth. Genomic DNA was extracted using the QIAsymphony DNA Midi kit (Qiagen, Hilden, Germany), followed by bisulfite treatment using the Zymo EZ-96 DNA methylation kit (Zymo Research, Irvine, California, USA). Genome-wide DNA methylation status for $>850 \mathrm{~K}$ CpG sites was analyzed using the Infinium MethylationEPIC BeadChip array (Illumina, San Diego, California, USA). Methylation data were acquired using the iScan system (Illumina) as idat files and processed by the minfi and ChAMP packages (https://bioconductor.org/biocLite.R) in R (V.3.4.0; www.R-project.org). The background was corrected using the NOOB method in the minfi package. ${ }^{25}$ Corrected data were normalized by BMIQ in the ChAMP package (V.2.8.9) ${ }^{26}$ The manifest file was annotated using 'IlluminaHumanMethylationEPICanno.ilm10b2.hg19'. We removed 11800 probes with detection $p$ values $>0.01$ in at least one sample, 3125 probes with a bead count $<3$ in at least $5 \%$ of samples and 2894 non-CpG targeting probes. Additionally, we filtered 17124 probes located on either the $\mathrm{X}$ or $\mathrm{Y}$ chromosome, 49 multihit probes, ${ }^{27}$ and 77589 single-nucleotide polymorphism-related probes using ChAMP. ${ }^{28}$ This yielded 754255 autosomal probes from 230 samples. We used the beta value $(\beta)$ for our analysis, representing the ratio of the methylated probe intensity and overall intensity (sum of methylated and unmethylated probe intensities). The cell composition 
of each cord blood sample (ie, 'Bcell', 'CD4T', 'CD8T', 'Gran', 'Mono', 'NK', and 'nRBC') was analyzed using 'FlowSorted.CordBlood.450k' in the minfi package. ${ }^{29} \mathrm{We}$ confirmed that the same sample had not been measured twice as clustering samples by using methylation levels of 1297 probes with a minor allele frequency of the target CpG site $>0.40 .^{30}$

\section{Statistical analysis}

Perinatal information was retrospectively obtained from medical records. The $\mathrm{p}$ value was adjusted to correct for multiple testing by controlling the expected proportion of false-positives among all discoveries (ie, false discovery rate) using the Benjamini-Hochberg method. Multiple testing were performed using the false discovery rate method, and thresholds of 0.05 and 0.01 were compared. Data are presented as the median (range) or number of cases (percentage). Continuous data were compared between groups using the Mann-Whitney U test. Categorical variables were analyzed using the $\chi^{2}$ test or Fisher's exact test. The trend for the number of abnormal values during OGTT was analyzed by Cochran-Armitage trend analysis. All tests were statistically analyzed by JMP software (V.15, SAS Institute), and $\mathrm{p}$ values $<0.05$ were considered as significant.

For the epigenome-wide association study, we assessed raw and normalized $\beta$ values from the 230 samples using principal component analysis to exclude outliers, which resulted in exclusion of eight samples (E-GDM: $n=2$, L-GDM: $n=3$, normal OGTT: $n=3$ ) from further analysis. Robust linear regression analysis was performed to examine the associations between DNA methylation and GDM, E-GDM, or L-GDM. Furthermore, we analyzed the DNA methylation between insulin and diet therapy group and diet group in GDM mothers. Cell composition, neonate sex, maternal age, maternal pregravid BMI, and experiment plate batch were included to analyses as covariates. The $p$ value was adjusted using the BenjaminiHochberg method to correct for multiple testing. An adjusted $\mathrm{p}<0.05$ was considered as significant.

\section{RESULTS}

Maternal and neonatal characteristics of GDM and normal OGTT

Maternal and neonatal characteristics of the GDM (E-GDM and L-GDM) and normal OGTT groups are shown in table 1. For metabolic parameters, FPG, 1-hour and 2-hour PG, and HOMA-IR were higher in the GDM group than in the normal OGTT group (all $\mathrm{p}<0.001$ ). However, mothers with GDM showed a lower IS $_{\text {OGTT }}$ and ISSI-2 relative to mothers with normal OGTT (both $\mathrm{p}<0.001$ ), whereas no difference was observed in terms of the insulinogenic index. The median HbAlc was $5.4 \%$

\begin{tabular}{|c|c|c|c|}
\hline Characteristic & GDM (n=162) & Normal OGTT $(n=60)$ & $P$ value \\
\hline Maternal age at delivery (years) & $36(26-47)$ & $36(30-45)$ & 0.43 \\
\hline Nulliparity & $102(63 \%)$ & $44(73 \%)$ & 0.16 \\
\hline Maternal pregravid BMI $\left(\mathrm{kg} / \mathrm{m}^{2}\right)$ & $20.5(16.6-32.9)$ & $19.7(16.4-24.8)$ & 0.0026 \\
\hline $\mathrm{HbA} 1 \mathrm{c}$ at first trimester (\%) & $5.0(3.7-6.2)$ & $5.2(4.5-5.9)$ & 0.0035 \\
\hline Plasma glucose of the antepartum $75 \mathrm{~g}$ OGTT (weeks) & $20(8-35)$ & $27(20-30)$ & $<0.0001$ \\
\hline $\mathrm{FPG}(\mathrm{mg} / \mathrm{dL})$ & $92(54-114)$ & $82(66-89)$ & $<0.0001$ \\
\hline $1 \mathrm{~h}-\mathrm{PG}(\mathrm{mg} / \mathrm{dL})$ & $161(88-223)$ & $141(93-175)$ & $<0.0001$ \\
\hline $2 \mathrm{~h}-\mathrm{PG}(\mathrm{mg} / \mathrm{dL})$ & $144(68-227)$ & $119(78-152)$ & $<0.0001$ \\
\hline |GI & $0.73(0.02-7.00)$ & $0.78(-1.50-4.83)$ & 0.97 \\
\hline ISSI-2 & $1.87(0.61-3.85)$ & $2.25(1.43-5.01)$ & $<0.0001$ \\
\hline HOMA-IR & $1.36(0.18-17.17)$ & $1.02(0.19-3.20)$ & 0.003 \\
\hline $\mathrm{IS}_{\mathrm{OG} T}$ & $5.81(1.14-18.46)$ & $7.76(1.90-27.55)$ & $<0.0001$ \\
\hline GW at delivery (weeks) & $39(37-41)$ & $39(37-41)$ & 0.66 \\
\hline Cesarean section delivery & $60(37 \%)$ & $20(33 \%)$ & 0.64 \\
\hline Neonatal sex (female) & $74(46 \%)$ & 27 (45\%) & 1.00 \\
\hline Birth weight (g) & 3022 (2352-3868) & 2965 (2226-3666) & 0.19 \\
\hline Macrosomia & $0(0)$ & $0(0)$ & - \\
\hline Umbilical artery $\mathrm{pH}$ & $7.31(7.13-7.44)$ & $7.32(7.21-7.43)$ & 0.033 \\
\hline Apgar score, $1 \mathrm{~min}$ & $9(7-10)$ & $9(7-10)$ & 0.57 \\
\hline Apgar score, $5 \mathrm{~min}$ & $9(7-10)$ & $9(8-10)$ & 0.63 \\
\hline
\end{tabular}

Data represent median (range) or $\mathrm{n}(\%)$.

BMI, body mass index; FPG, fasting plasma glucose; GDM, gestational diabetes mellitus; GW, gestational weeks; HOMA-IR, homeostasis model assessment for insulin resistance; IGI, insulinogenic index; IS ${ }_{\mathrm{OGT}}$, insulin sensitivity index from OGTT; ISSI-2, insulin secretion sensitivity index-2; OGTT, oral glucose tolerance test; PG, plasma glucose. 
Table 2 Comparison of maternal and neonatal characteristics between pregnant Japanese women with GDM and normal OGTT

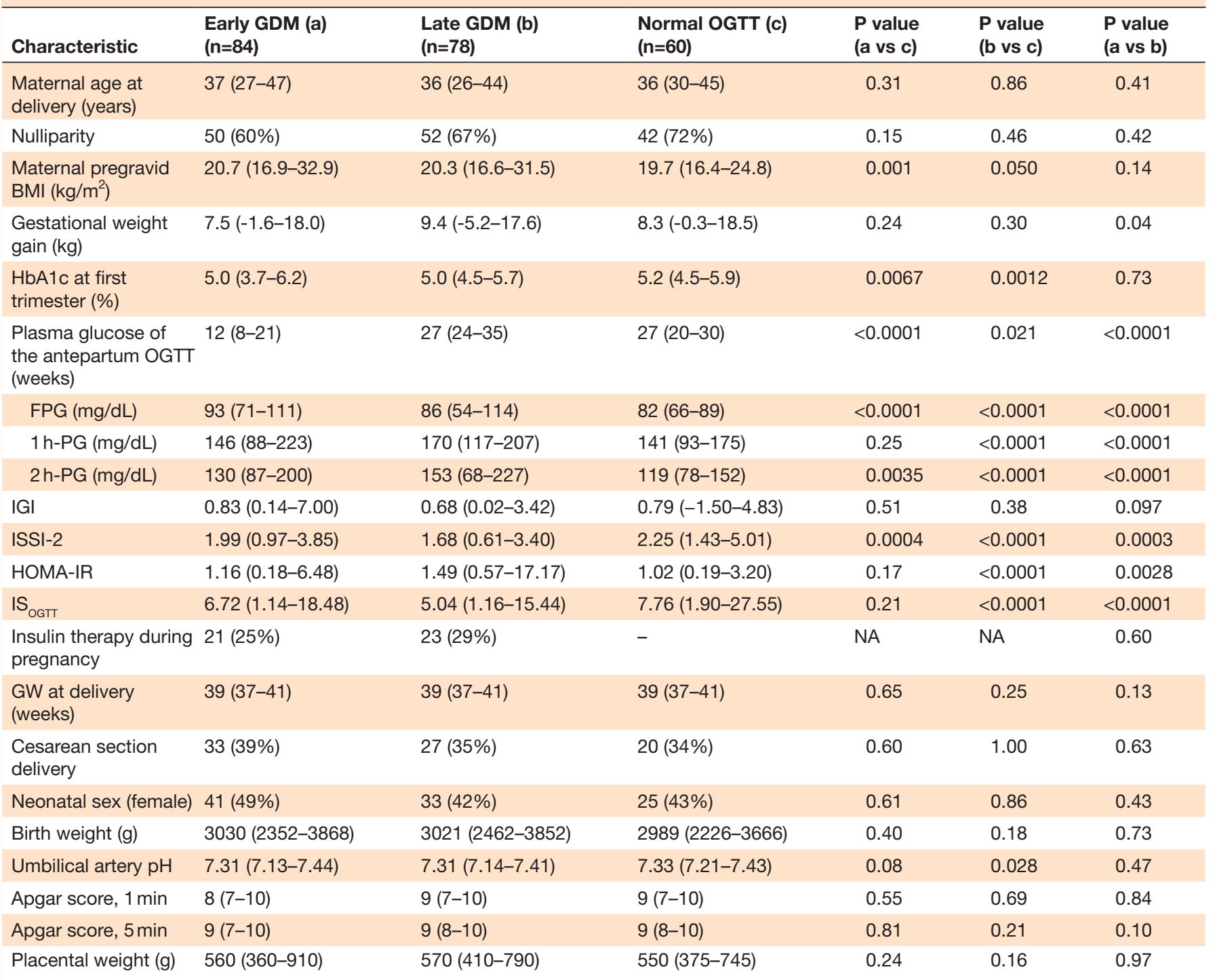

Data represent median (range) or $\mathrm{n}(\%)$.

BMI, body mass index; FPG, fasting plasma glucose; GDM, gestational diabetes mellitus; GW, gestational weeks; HOMA-IR, homeostasis model assessment for insulin resistance; IGI, insulinogenic index; IS ${ }_{\mathrm{OG} \text {, }}$ insulin sensitivity index from OGTT; ISSI-2, insulin secretion sensitivity index-2; OGTT, oral glucose tolerance test; PG, plasma glucose.

(range: $4.6 \%-6.4 \%$ ), and the median glycoalbumin (GA) was $13.1 \%$ (range: $9.7 \%-16.0 \%$ ) at the third trimester in the GDM group. However, because none of the patients had HbAlc $\geq 5.8 \%$ and $\mathrm{GA} \geq 15.8 \%$ at the third trimester, it was considered that the glucose levels were well controlled in all GDM patients.

\section{Maternal and neonatal characteristics of early GDM and late GDM}

The maternal and neonatal characteristics of the E-GDM and L-GDM groups are shown in table 2. Regarding metabolic features, FPG, ISSI-2, and IS ${ }_{\text {OGTT }}$ in E-GDM were significantly higher than those in L-GDM; however, $1 \mathrm{~h}$ PG, $2 \mathrm{~h}$ PG, and HOMA-IR in E-GDM were significantly lower than those in L-GDM. There were no significant differences in perinatal outcomes (ie, gestational week at delivery, birth weight, $\mathrm{CS}$ incidence, umbilical artery $\mathrm{pH}$, Apgar scores, and placental weight) or in the incidence of insulin therapy during pregnancy between the groups. However, gestational weight gain in E-GDM was significantly lower than that in L-GDM.

\section{Cord blood epigenome-wide association study for GDM}

In DNA methylation analysis, newborn sex, gestational age (in days), and estimated proportions of B cells, CD8 + $\mathrm{T}$ cells, CD4 + T cells, natural killer cells, monocytes, and nucleated red blood cells in the cord blood were included in multivariate linear regression analysis as covariates. There was no significant association between DNA methylation values and GDM compared with in the normal OGTT group (figure 1). Furthermore, in subgroup analysis, there were no significant association between DNA 


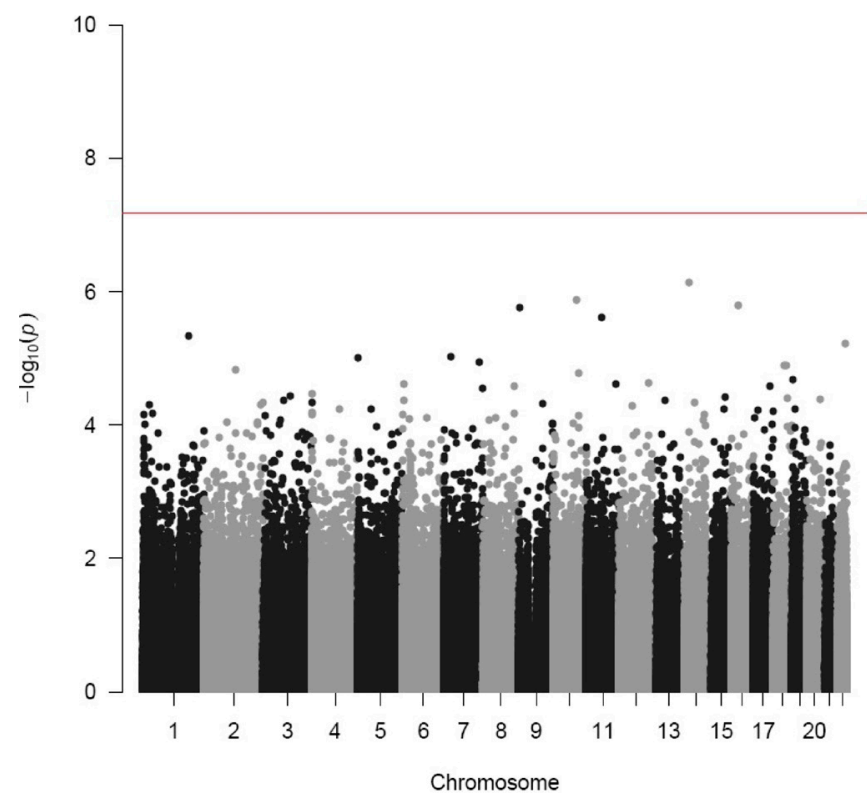

Figure 1 Comparison of DNA methylation between GDM and normal OGTT groups. The $\mathrm{x}$-axis shows mean DNA methylation differences in GDM compared with normal OGTT, and the $y$-axis shows the $-\log _{10}$ of the $p$ value for each CpG (5'-Cytosine-phosphate-Guanine-3) site and represents the strength of association. The color of each dot represents the $\mathrm{p}$ value adjusted, as shown in the scale bar. GDM, gestational diabetes mellitus; OGTT, oral glucose tolerance test.

methylation values and E-GDM or L-GDM compared with in the normal OGTT group and between DNA methylation values and in the E-GDM group compared with in the L-GDM group (figure 2A-C). We classified the GDM group into insulin and diet therapy group and diet group and found that there was no significant difference in the DNA methylation level in each group compared with that in the normal OGTT group (figure 2D,E).

\section{DISCUSSION}

We found no significant difference in umbilical cord blood DNA methylation between GDM and normal OGTT. Moreover, patients with GDM classified as E-GDM and L-GDM based on Japan Society of Obstetrics and Gynecology criteria showed no differences in the DNA methylation profiles among groups.

Epigenetic modifications associated with GDM are thought to influence the susceptibility to metabolic disorders in offspring. ${ }^{4-12}$ An overview of genome-wide methylation analyses of GDM using cord blood obtained from earlier empirical studies is shown in table 3. In all studies, epigenetic changes were detected in metabolic mechanisms-related genes; however, in those reports, the maternal glycemic state during pregnancy and fetal information were not described in detail, and the studies included small cohorts. Additionally, the criteria for diagnosing GDM differs from IADPSG criteria in many reports. In our study cohort, all mothers with GDM underwent dietary management, with self-monitoring of blood glucose and insulin administration initiated when dietary modification did not achieve glycemic control. We also excluded neonates who were born at preterm or developed neonatal asphyxia, and we adjusted cell counts based on reference data. ${ }^{31}$ Although various causes of epigenetic changes in offspring have been reported, ${ }^{32} 33$ the findings from our cohort indicate that DNA methylation in cord blood is not altered when mothers with GDM take proper precautions during pregnancy. However, the absolute methylation rates between our study and other studies were distinct (data not shown) in some candidate loci that previous studies identified as differentially methylated in GDM. For example, the average global methylation levels differed by 3\%-4\% between our study and that by Antoun et al. ${ }^{12}$ Furthermore, absolute methylation rates at 8 out of the top 10 differentially methylated sites between GDM and non-GDM, reported by Antoun et al ${ }^{12}$ differed by $5 \%-15 \%$ between our subjects and those in the study by Antoun et al regardless of the presence of GDM. These differences in absolute methylation rates between studies were also observed at six CpG sites that were identified by Howe $e t a l^{44}$ in a meta-analysis as being differentially methylated in cord blood cells from infants born to GDM mothers. These mismatches might be a result of effects of ethnicity. Genetic variation with ethnicity may influence the effects of GDM on infant methylome. Further analysis introducing standardized methods for cell collection and data normalization could help understand accurate universal effects of GDM on infant methylome.

According to our previous data, there were no difference in perinatal outcomes (ie, preterm delivery, hypertensive disorder of pregnancy, CS, macrosomia, and insulin therapy) between E-GDM and L-GDM, ${ }^{15}$ although others studies showed that E-GDM had poor perinatal prognosis. Moreover, when patients were diagnosed with GDM in early pregnancy and started treatment, as their gestational weight gain was limited, it was expected that fetal growth during pregnancy would be decreased. The previous reports showed that treatment of mild GDM during pregnancy did not reduce childhood obesity or metabolic dysfunctions in the offspring. ${ }^{35} 36$ Therefore, Hagiwara et $a l^{14}$ reported that the therapeutic interventions for E-GDM were not as effective as those for L-GDM. However, another study reported that the incidence of preterm birth in women with E-GDM who were followed up without treatment and had abnormal OGTT results at 24-28 gestational weeks was significantly higher than that in women with E-GDM who were followed up without treatment and had normal OGTT results at 24-28 gestational weeks $(p=0.01) .{ }^{37}$ According to our previous report, management of E-GDM may be an appropriate option. ${ }^{15}$ In the report by Antoun $e t a l^{12}$ using the IADPSG as a diagnostic criterion for GDM, only L-GDM diagnosed after 24 weeks of gestation was recruited. Although Antoun et al identified GDM-associated methylation changes in cord blood cells from larger populations than ours, it was revealed that there was no significant methylation 

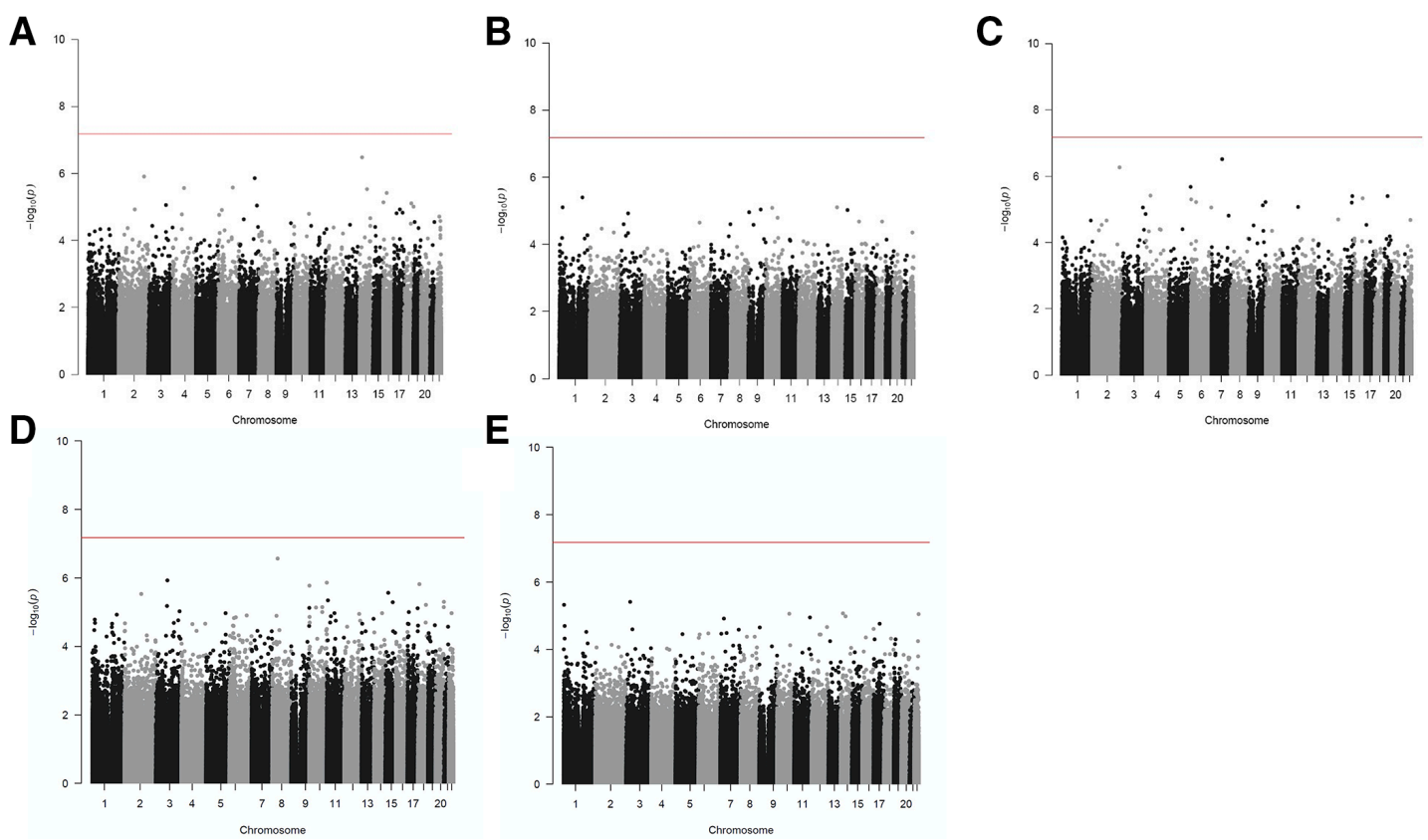

Figure 2 Subgroup analysis of DNA methylation. (A) Comparison of DNA methylation between E-GDM and normal OGTT groups. The $x$-axis shows the mean DNA methylation differences in E-GDM compared with normal OGTT. (B) Comparison of DNA methylation between L-GDM and normal OGTT groups. The $x$-axis shows the mean DNA methylation differences in LGDM compared with normal OGTT. (C)Comparison of DNA methylation between E-GDM and L-GDM groups. The $\mathrm{x}$-axis shows the mean DNA methylation differences in E-GDM compared with L-GDM. (D) Comparison of DNA methylation between the insulin and diet therapy and normal OGTT groups. The x-axis shows the mean DNA methylation differences in the insulin and diet group compared with those in the normal OGTT group. (E) Comparison of DNA methylation between the diet therapy and normal OGTT groups. The $\mathrm{x}$-axis shows the mean DNA methylation differences in the diet therapy group compared with those in the normal OGTT group. The $y$-axis shows the $-\log _{10}$ of the $p$ value for each 5 '-Cytosine-phosphate-Guanine-3 (CpG) site and representing the strength of association. The color of each dot represents the $\mathrm{p}$ value adjusted, as shown in the scale bar. E-GDM, early gestational diabetes mellitus; L-GDM, late gestational diabetes mellitus; OGTT, oral glucose tolerance test.

changes between E-GDM or L-GDM or normal OGTT in this study. The early diagnosis and therapeutic intervention of GDM may have caused the difference in results from studies involving L-GDM, which have a longer period of no intervention from onset to diagnosis.
This study had several limitations. First, our study cohort was relatively small. According to user-friendly tool for comprehensive power estimation for epigenome wide association studies (pwrEWAS), the mean statistical power required to detect a $20 \%$ methylation difference

Table 3 Overview of genome-wide methylation analyses of GDM using cord blood in published studies

\begin{tabular}{|c|c|c|c|c|c|c|}
\hline Study & $\begin{array}{l}\text { Number of } \\
\text { differentially } \\
\text { methylated CpG } \\
\text { sites }\end{array}$ & Method & $\begin{array}{l}\text { GDM } \\
(n)\end{array}$ & $\begin{array}{l}\text { Non-GDM } \\
(n)\end{array}$ & $\begin{array}{l}\text { Criteria for diagnosing } \\
\text { GDM }\end{array}$ & Ethnicity \\
\hline Ruchat et al ${ }^{9}$ & 7855 & $450 \mathrm{~K}$ & 30 & 14 & WHO & Caucasian \\
\hline Finer et $a l^{4}$ & 1418 & $450 \mathrm{~K}$ & 21 & 28 & WHO & South Asian \\
\hline Haertle et $a l^{5}$ & 65 & $450 \mathrm{~K}$ & 68 & 64 & ADA & Caucasian \\
\hline Kang et $a l^{6}$ & 200 & EPIC & 8 & 8 & IADPSG & Chinese \\
\hline Awamleh, et al ${ }^{11}$ & 99 & $450 \mathrm{~K}$ & 16 & 26 & Carpenter-Coustan & Caucasian \\
\hline Antoun et $a l^{12}$ & 242 & EPIC & 159 & 383 & IADPSG & Various \\
\hline This study & 0 & EPIC & 162 & 60 & IADPSG & Japanese \\
\hline
\end{tabular}

ADA, American Diabetes Association; CpG, 5'-Cytosine-phosphate-Guanine-3; EPIC, Infinium Human Methylation EPIC BeadChip; GDM, gestational diabetes mellitus; IADPSG, International Association of Diabetes and Pregnancy Study Group; 27K, human methylation $27 \mathrm{~K}$ bead chip; $450 \mathrm{~K}$, human methylation $450 \mathrm{~K}$ bead chip. 
between GDM and normal OGTT groups with our sample size was 0.779 . The $2 \%$ difference that we identified at the two CpG sites is a minor difference with respect to the size of our dataset, as the statistical power to detect such difference was 0.218 . However, our sample size was larger than those reported previously. ${ }^{4-12}$ In a previous report on GDM diagnosed by a single criterion, the power of the largest epigenome-wide association study was 0.143 in case of a $2 \%$ difference. Therefore, the power of our results was stronger than that of the previous report, and the present study is the largest genome-wide association study of GDM diagnosed by a single criterion. A recent large cohort study reported two upregulated genes as associated with maternal GDM ${ }^{34}$ however, because GDM was diagnosed according to several inconsistent criteria in the previous cohort, we considered that those results may not be entirely accurate. Furthermore, we excluded neonates born at preterm or who developed neonatal asphyxia, as these factors may have affected the DNA methylation of umbilical blood samples. Therefore, our results may be more meaningful. Second, although our results were different from the previous reports that suggested epigenetic changes in the metabolic genes, we could not determine whether the difference was due to better control of GDM during pregnancy. As Asian women, including Japanese women, have impaired beta cell compensation compared with Caucasian and Hispanic women, ${ }^{38} 39$ the mechanism of GDM development might be different between Japanese and other ethnicities. Therefore, these results might reflect a condition unique to Asian patients with GDM. Furthermore, our patients were older than the patients enrolled in previous studies and the $\mathrm{HbAlc}$ level in the first trimester in the normal OGTT group was higher than that in the GDM group. We could not determine whether the differences in maternal characteristics affected the results or not. However, we hypothesized that categorizing and managing E-GDM and L-GDM may have had a positive impact on DNA methylation of cord blood.

Our genome-wide methylation analysis revealed no differences in the methylation level in cord blood from offspring born to mothers with GDM relative to in those with Normal OGTT among not only L-GDM but E-GDM. Further studies are required to determine whether these results are associated with long-term outcomes in offspring.

\section{Author affiliations}

'Department of Obstetrics and Gynecology, Keio University School of Medicine, Shinjuku-ku, Tokyo, Japan

${ }^{2}$ Department of Maternal-Fetal Biology, National Research Institute for Child Health and Development, Setagaya-ku, Tokyo, Japan

${ }^{3}$ Division of Fetal Development, National Research Institute for Child Health and Development, Setagaya-ku, Tokyo, Japan

${ }^{4}$ Department of Medical Genetics and Genomics, Kitasato University Graduate School of Medical Sciences, Sagamihara, Kanagawa, Japan

${ }^{5}$ Department of Internal Medicine, Keio University School of Medicine, Shinjuku-ku, Tokyo, Japan

${ }^{6}$ Department of Pediatrics, Keio University School of Medicine, Shinjuku-ku, Tokyo, Japan
Acknowledgements The authors would like to thank the medical staff in the perinatal care unit of Keio University Hospital for excellent patient care, and the staff of the Department of Maternal-Fetal Biology, National Research Institute for Child Health and Development, and special thanks to Professor Atsushi Tajima, Department of Bioinformatics and Genomics, Graduate School of Advanced Preventive Medical Sciences, Kanazawa University, for his support. We thank Editage (www.editage.jp) for English language editing.

Contributors YK and TK collected the data, performed statistical analyses, wrote the manuscript, contributed to the discussion, and reviewed/edited the manuscript: $\mathrm{KM}$ contributed to the discussion and wrote and reviewed/edited the manuscript; AH collected the data; MTam, KHas, SI, DO, YS, MH, MTan, and KHat contributed to the discussion and reviewed/edited the manuscript; and all authors approved the final manuscript for publication. TK and KHat are responsible for the overall content as the guarantor.

Funding This study was supported by the Japan Agency for Medical Research and Development grant number 18ek0109278h0002, 18ek0109290h0002, and 18mk0102093s0402, and by the Japan Society for the Promotion of Science KAKENHI grant number 17K19535 and 19K09761.

Competing interests None declared.

\section{Patient consent for publication Not applicable.}

Ethics approval This study involves human participants and was approved by Keio University School of Medicine Ethics Committee (nos. 20100154, approved 27 September 2010; 20110321, approved 9 March 2012; 20150103, approved 23 June $2015 ; 20150168$, approved 31 July 2015) and the institutional review board of National Research Institute for Child Health and Development (no. 234, approved 5 March 2012) and was performed in accordance with the ethical standards outlined in the 1964 Declaration of Helsinki and its later amendments. All study participants provided informed consent. Participants gave informed consent to participate in the study before taking part

Provenance and peer review Not commissioned; externally peer reviewed.

Data availability statement Data are available on reasonable request. The data set supporting the results of this article are available in the Gene Expression Omnibus repository (GSE122086, https://www.ncbi.nlm.nih.gov/geo/query/acc. cgi?acc=GSE122086; and GSE122288, https://www.ncbi.nlm.nih.gov/geo/query/ acc.cgi?acc=GSE122288https://www.ncbi.nlm.nih.gov/geo/query/acc.cgi?acc= GSE122288).

Open access This is an open access article distributed in accordance with the Creative Commons Attribution Non Commercial (CC BY-NC 4.0) license, which permits others to distribute, remix, adapt, build upon this work non-commercially, and license their derivative works on different terms, provided the original work is properly cited, appropriate credit is given, any changes made indicated, and the use is non-commercial. See: http://creativecommons.org/licenses/by-nc/4.0/.

\section{ORCID iDs}

Tomoko Kawai http://orcid.org/0000-0001-8137-0334

Yoshifumi Saisho http://orcid.org/0000-0002-6071-4393

\section{REFERENCES}

1 Fetita L-S, Sobngwi E, Serradas P, et al. Consequences of fetal exposure to maternal diabetes in offspring. J Clin Endocrinol Metab 2006;91:3718-24.

2 Dabelea D, Hanson RL, Lindsay RS, et al. Intrauterine exposure to diabetes conveys risks for type 2 diabetes and obesity: a study of discordant sibships. Diabetes 2000;49:2208-11.

3 Clausen TD, Mathiesen ER, Hansen T, et al. Overweight and the metabolic syndrome in adult offspring of women with diet-treated gestational diabetes mellitus or type 1 diabetes. J Clin Endocrinol Metab 2009;94:2464-70.

4 Finer S, Mathews C, Lowe R, et al. Maternal gestational diabetes is associated with genome-wide DNA methylation variation in placenta and cord blood of exposed offspring. Hum Mol Genet 2015;24:3021-9.

5 Haertle L, El Hajj N, Dittrich M, et al. Epigenetic signatures of gestational diabetes mellitus on cord blood methylation. Clin Epigenetics 2017;9:28

6 Kang J, Lee C-N, Li H-Y, et al. Genome-Wide DNA methylation variation in maternal and cord blood of gestational diabetes population. Diabetes Res Clin Pract 2017;132:127-36.

7 Moen G-H, Sommer C, Prasad RB, et al. Mechanisms in endocrinology: epigenetic modifications and gestational diabetes: 
a systematic review of published literature. Eur $\mathrm{J}$ Endocrinol 2017;176:R247-67.

8 Quilter CR, Cooper WN, Cliffe KM, et al. Impact on offspring methylation patterns of maternal gestational diabetes mellitus and intrauterine growth restraint suggest common genes and pathways linked to subsequent type 2 diabetes risk. Faseb J 2014;28:4868-79.

9 Ruchat S-M, Houde A-A, Voisin G, et al. Gestational diabetes mellitus epigenetically affects genes predominantly involved in metabolic diseases. Epigenetics 2013;8:935-43.

10 Wu P, Farrell WE, Haworth KE, et al. Maternal genome-wide DNA methylation profiling in gestational diabetes shows distinctive disease-associated changes relative to matched healthy pregnancies. Epigenetics 2018;13:122-8.

11 Awamleh Z, Butcher DT, Hanley A, et al. Exposure to gestational diabetes mellitus (GDM) alters DNA methylation in placenta and fetal cord blood. Diabetes Res Clin Pract 2021;174:108690.

12 Antoun E, Kitaba NT, Titcombe P, et al. Maternal dysglycaemia, changes in the infant's epigenome modified with a diet and physical activity intervention in pregnancy: secondary analysis of a randomised control trial. PLoS Med 2020;17:e1003229.

13 Minakami H, Maeda T, Fujii T, et al. Guidelines for obstetrical practice in Japan: Japan Society of obstetrics and gynecology (JSOG) and Japan association of obstetricians and Gynecologists (JAOG) 2014 edition. J Obstet Gynaecol Res 2014;40:1469-99.

14 Hagiwara Y, Kasai J, Nakanishi S, et al. Should the IADPSG criteria be applied when diagnosing early-onset gestational diabetes? Diabetes Res Clin Pract 2018;140:154-61.

15 Tamagawa M, Kasuga Y, Saisho Y, et al. Predictors of later insulin therapy for gestational diabetes diagnosed in early pregnancy. Endocr J 2021;68:1321-8.

16 Sweeting AN, Ross GP, Hyett J, et al. Gestational diabetes mellitus in early pregnancy: evidence for poor pregnancy outcomes despite treatment. Diabetes Care 2016;39:75-81.

17 Usami T, Yokoyama M, Ueno M, et al. Comparison of pregnancy outcomes between women with early-onset and late-onset gestational diabetes in a retrospective multi-institutional study in Japan. J Diabetes Investig 2020;11:216-22.

18 Feghali MN, Abebe KZ, Comer DM, et al. Pregnancy outcomes in women with an early diagnosis of gestational diabetes mellitus. Diabetes Res Clin Pract 2018;138:177-86.

19 Bashir M, Baagar K, Naem E, et al. Pregnancy outcomes of early detected gestational diabetes: a retrospective comparison cohort study, Qatar. BMJ Open 2019;9:e023612.

20 Matsuda M, DeFronzo RA. Insulin sensitivity indices obtained from oral glucose tolerance testing: comparison with the euglycemic insulin clamp. Diabetes Care 1999;22:1462-70.

21 Matthews DR, Hosker JP, Rudenski AS, et al. Homeostasis model assessment: insulin resistance and beta-cell function from fasting plasma glucose and insulin concentrations in man. Diabetologia 1985;28:412-9.

22 Kosaka K, Hagura R, Kuzuya T, et al. Insulin secretory response of diabetics during the period of improvement of glucose tolerance to normal range. Diabetologia 1974;10:775-82.
23 Retnakaran R, Qi Y, Goran MI, et al. Evaluation of proposed oral disposition index measures in relation to the actual disposition index. Diabet Med 2009;26:1198-203.

24 Kasuga $\mathrm{Y}$, Kawai T, Miyakoshi K, et al. Epigenetic changes in neonates born to mothers with gestational diabetes mellitus may be associated with neonatal hypoglycaemia. Front Endocrinol 2021:12:690648.

25 Aryee MJ, Jaffe AE, Corrada-Bravo H, et al. Minfi: a flexible and comprehensive Bioconductor package for the analysis of Infinium DNA methylation microarrays. Bioinformatics 2014;30:1363-9.

26 Morris TJ, Butcher LM, Feber A, et al. Champ: 450k CHIP analysis methylation pipeline. Bioinformatics 2014;30:428-30.

27 Nordlund J, Bäcklin CL, Wahlberg P, et al. Genome-Wide signatures of differential DNA methylation in pediatric acute lymphoblastic leukemia. Genome Biol 2013;14:r105.

28 Zhou W, Laird PW, Shen H. Comprehensive characterization, annotation and innovative use of Infinium DNA methylation BeadChip probes. Nucleic Acids Res 2017;45:e22.

29 Bakulski KM, Feinberg Jl, Andrews SV, et al. Dna methylation of cord blood cell types: applications for mixed cell birth studies. Epigenetics 2016;11:354-62.

30 Peters TJ, Buckley MJ, Statham AL, et al. De novo identification of differentially methylated regions in the human genome. Epigenetics Chromatin 2015;8:6.

31 Shiwa Y, Hachiya T, Furukawa R, et al. Adjustment of cell-type composition minimizes systematic bias in blood DNA methylation profiles derived by DNA collection protocols. PLOS One 2016;11:e0147519

32 Girchenko P, Lahti J, Czamara D, et al. Associations between maternal risk factors of adverse pregnancy and birth outcomes and the offspring epigenetic clock of gestational age at birth. Clin Epigenetics 2017;9:49.

33 Kawai T, Yamada T, Abe K, et al. Increased epigenetic alterations at the promoters of transcriptional regulators following inadequate maternal gestational weight gain. Sci Rep 2015;5:14224.

34 Howe CG, Cox B, Fore R, et al. Maternal gestational diabetes mellitus and newborn DNA methylation: findings from the pregnancy and childhood epigenetics Consortium. Diabetes Care 2020;43:98-105.

35 Gillman MW, Oakey H, Baghurst PA, et al. Effect of treatment of gestational diabetes mellitus on obesity in the next generation. Diabetes Care 2010;33:964-8.

36 Landon MB, Rice MM, Varner MW, et al. Mild gestational diabetes mellitus and long-term child health. Diabetes Care 2015;38:445-52.

37 Nakanishi S, Aoki S, Kasai J, et al. High probability of false-positive gestational diabetes mellitus diagnosis during early pregnancy. BMJ Open Diabetes Res Care 2020;8:e01234.

38 Sattar N, Gill JMR. Type 2 diabetes in migrant South Asians: mechanisms, mitigation, and management. Lancet Diabetes Endocrinol 2015;3:1004-16.

39 Tutino GE, Tam WH, Yang X, et al. Diabetes and pregnancy: perspectives from Asia. Diabet Med 2014;31:302-18. 